\title{
A NEW IMPLICIT METHOD FOR SURFACE SEGMENTATION BY MINIMAL PATHS IN 3D IMAGES
}

\author{
Roberto Ardon ${ }^{1,2}$ \\ Laurent D. Cohen ${ }^{2}$ (corresponding author: Phone: +331440546 78, Fax: +33144 \\ 0545 99, Email: cohen@ceremade.dauphine.fr) \\ Anthony Yezzi $^{3}$ \\ ${ }^{1}$ MEDISYS-Philips France, 51, rue Carnot, 92156 Suresnes, France, \\ 2 CEREMADE-Université Paris Dauphine, Place du Maréchal de Lattre de Tas- \\ signy, 75775 Paris Cedex 16, France \\ ${ }^{3}$ Georgia Institute of Technology, Atlanta, GA, USA, \\ roberto.ardon@philips.com, cohen@ceremade.dauphine.fr, ayezzi@ece.gatech.edu.
}

\begin{abstract}
We introduce a novel implicit approach for single object segmentation in 3D images. The boundary surface of this object is assumed to contain two known curves (the constraining curves), given by an expert. The aim of our method is to find the wanted surface by exploiting as much as possible the information given in the supplied curves and in the image. As for active surfaces, we use a cost potential which penalizes image regions of low interest (most likely areas of low gradient or too far from the surface to be extracted). In order to avoid local minima, we introduce a new partial differential equation and use its solution for segmentation. We show that the zero level set of this solution contains the constraining curves as well as a set of paths joining them. We present a fast implementation which has been successfully applied to $3 \mathrm{D}$ medical and synthetic images.
\end{abstract}

keywords: Image segmentation, Active contours, Minimal Paths, Level Set method, Stationary Transport Equation.

\section{Introduction}

The common use of deformable models, introduced by Kass et al. [12], in 2D and 3D image segmentation consists in introducing an initial object in the image and deforming it until it reaches a desired target. In [10], Cohen and Kimmel present a segmentation approach in 2D where the final state of the active curve stands for the global minimum of an image dependent energy. Their model only requires two points located on the boundary to be segmented as additional information. Unfortunately their approach cannot be extended to find the global minimum for an active surface in a $3 \mathrm{D}$ image. 
In this work, we focus on a novel approach for 3D single object segmentation where the resulting surface globally minimizes a given energy. Our aim is to generate a surface that contains a couple of 'constraining' curves $\left(\Gamma_{1}\right.$ and $\left.\Gamma_{2}\right)$ and which is also a segmentation of a target object. $\Gamma_{1}$ and $\Gamma_{2}$ are supposed to be traced by an expert on the surface to be segmented. Our approach is based on implicitly generating a surface that contains the set of paths globally minimizing an image energy and connecting $\Gamma_{1}$ and $\Gamma_{2}$. Moreover, the constraining curves are the only input for the initialization of our model. The paths linking $\Gamma_{1}$ and $\Gamma_{2}$ are globally minimal with respect to an energy of the form $\int_{\Gamma} \mathcal{P}$. If the incremental cost $\mathcal{P}$ is chosen to take lower values on the contours of the 3D image, in particular on the surface of the object to be extracted, global minimal paths will help finding the boundary of the object (see [10]). This fact has been exploited in previous work [3], where a network of a finite number of minimal paths was computed between the two constraining curves and then extended, by means of interpolation, to a segmentation surface of the object.

Although this approach gave good results, particularly in ultrasound 3D images, the topology of the network was often problematic (paths tend to merge and only few points of $\Gamma_{1}$ are to be reached, see figure 1.d) considerably complicating the generation of the segmenting surface and in the worst cases leading to bad segmentations (figure 6.b).

Although based on similar ideas, our model is more than an extension of the network approach. The surface generated by our algorithm is completely composed of globally minimal paths, and in particular, it contains all the minimal paths of the network introduced in [3] (no explicit computation of minimal paths is needed nor any interpolation method). Indeed, by solving a stationary transport equation of the form: $\nabla \Psi \cdot \nabla U+G(\Psi)=0$, where $G$ is a function such that $G(0)=0, \Psi$ is the unknown, and $U$ is the action map defined by Eqn. (15). We show in section 3 that $\Psi$ is such that: any minimal path between the constraining curves is contained in its zero level set (further noted $\Psi^{-1}(\{0\})$ ). More generally, we prove that this set is only composed of minimal paths: if a point belongs to $\Psi^{-1}(\{0\})$ then the globally minimal path joining this point to $\Gamma_{1}$ is contained as well in $\Psi^{-1}(\{0\})$. This property is the key point explaining the good performance of our algorithm. In section 4 we give some results obtained by our method on synthetic and real data.

As an illustration of our problem, we give in figures 1 an example of the user input to our algorithm. Background on active contours, minimal paths in $2 \mathrm{D}$ and $3 \mathrm{D}$ images and the path network can be found in $[12,10,3]$. 


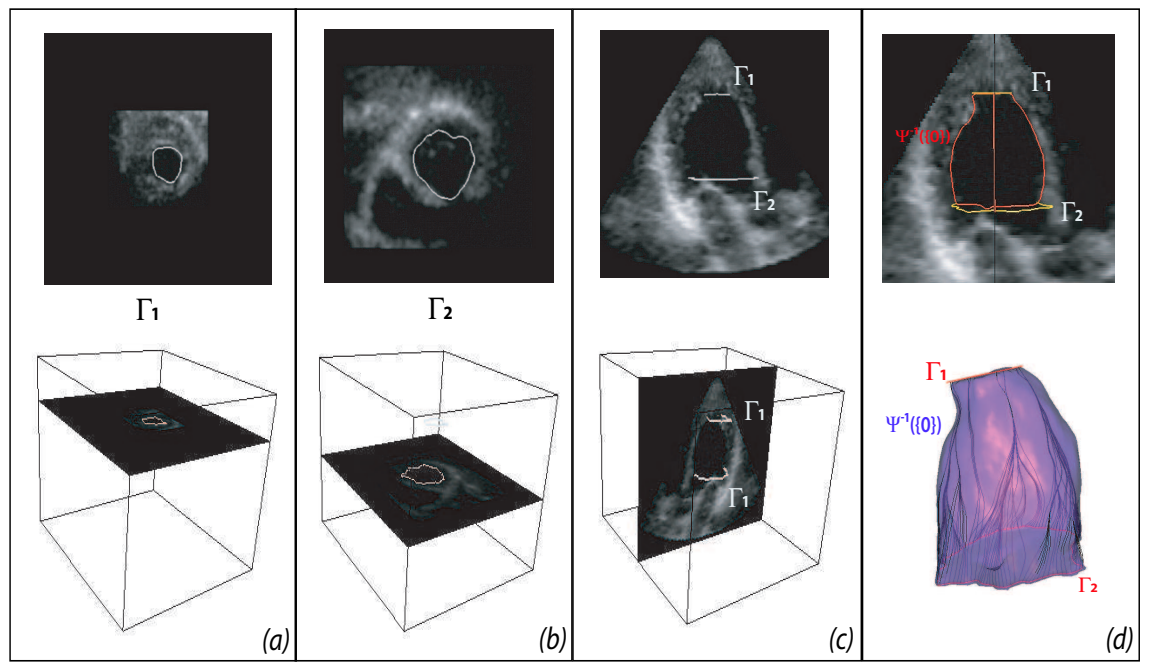

Figure 1: 3D ultrasound volume of a left ventricle: (a) and (b) show the two parallel slices where the user given curves $\Gamma_{1}$ and $\Gamma_{2}$ are drawn. (c) shows a slice perpendicular to the curves in order to show their position with respect to the ventricle. Finally (d) shows the surface containing the constraint curves obtained with our approach. In the upper position we have shown the intersection of the zero level set of $\Psi$ with a vertical plane. In the lower position we have traced some minimal paths between the two constraining curves and a $3 \mathrm{D}$ representation of the zero level set, the minimal paths are traced on this surface.

\section{Minimal Paths}

\subsection{Global minimal paths between two points}

Cohen and Kimmel give in [10] a method to find the global minimal path, connecting two points $p_{1}$ and $p_{2}$, with respect to a given cost function $\mathcal{P}>0$, also called potential function. In other words, they find the global minimum of the geodesic active contour's energy

$$
E(\mathcal{C})=\int_{0}^{L} \mathcal{P}(\mathcal{C}(s)) d s
$$

when imposing to the curve its two end points. $L$ is the length of the curve and $s$ is the arclength. The potential function $\mathcal{P}$ is defined on the image domain and corresponds to the features we want the curve to follow. For example, if $I$ is the image, with $\mathcal{P}=I$, the curve will follow dark structures. With $\mathcal{P}=\frac{1}{1+\|\nabla I\|^{2}}$, the curve will extract high values of the gradient, and that corresponds to contours. 
Notice that in the special case where $\mathcal{P}$ is a constant, the energy is proportional to the length of the curve, and we are looking for minimal geodesics.

Authors of [10] show that a globally minimal curve for equation (1) is obtained by following the opposite gradient direction on the minimal action map $U_{p_{1}}$, defined by

$$
U_{p_{1}}(q)=\inf _{\mathcal{C}(0)=p_{1}, \mathcal{C}(L)=q}\left\{\int_{0}^{L} \mathcal{P}(\mathcal{C}(s) d s\} .\right.
$$

The minimal path between $p_{2}$ and $p_{1}$ is thus obtained by solving the problem:

$$
\frac{d C}{d s}(s)=-\nabla U_{p_{1}}(C(s)) \text { with } C(0)=p_{2} .
$$

A proof of this will be given in Theorem 4. In order to compute $U_{p_{1}}$, Cohen and Kimmel [10] use the fact that this map is solution of the Eikonal equation ([5]):

$$
\left\|\nabla U_{p_{1}}\right\|=\mathcal{P} \text { and } U_{p_{1}}\left(p_{1}\right)=0 .
$$

A rigorous proof can be found in [5, 14]. Let us however give some ideas to understand this equation. First, notice that when $\mathcal{P}=1, U_{p_{1}}(p)$ is the minimal length of a path between $p$ and $p_{1}$. Thus $U_{p_{1}}$ is equal to the distance to $p_{1}$, and this is known that a distance map satisfies $\left\|\nabla U_{p_{1}}\right\|=1$. In the general case, $U_{p_{1}}$ can be seen as a weighted distance to $p_{1}$. Second, let us consider the level sets of $U_{p_{1}}$, and call $\mathcal{L}(t)$ the curve(s) on which $U_{p_{1}}=t$. Now assume $\delta$ is a small positive number, and $p$ is a point on $\mathcal{L}(t+\delta)$. A minimal path between $p_{1}$ and $p$ will cross $\mathcal{L}(t)$ at some point $q$. By definition of the two level sets, the energy of the whole path $t+\delta$ is equal to the energy of the path between $p_{1}$ and $q$, which is $t$, plus the energy of the small path between $p$ and $q$. Admitting that when $\delta$ gets very small, this small path will be close to a segment of length $l$, and normal to both level sets, that is in the direction of $\nabla U_{p_{1}}$, we have $T+\delta=t+l \mathcal{P}(p)$. This means that the local distance $l$ between the two level sets is equal to $\frac{\delta}{\mathcal{P}(p)}$. From these remarks, we can understand that the curves $\mathcal{L}(t)$ satisfy the evolution equation $\frac{\partial \mathcal{L}(s, t)}{\partial t}=\frac{1}{\mathcal{P}} \vec{n}(s, t)$, where $\vec{n}(s, t)$ is the unit normal vector to the curve $\mathcal{L}(., t)$. The last idea is that by differentiation of the identity $U_{p_{1}}(\mathcal{L}(s, t))=t$ with respect to $t$, we have $\nabla U_{p_{1}} \cdot \frac{\partial \mathcal{L}(s, t)}{\partial t}=1$. Replacing $\frac{\partial \mathcal{L}(s, t)}{\partial t}$ by $\frac{1}{\mathcal{P}} \vec{n}(s, t)$ and noticing that the normal to level sets of $U_{p_{1}}$ is the normalized gradient vector $\frac{\nabla U_{p_{1}}}{\left\|\nabla U_{p_{1}}\right\|}$, we have $\nabla U_{p_{1}} \cdot \frac{1}{\mathcal{P}} \frac{\nabla U_{p_{1}}}{\left\|\nabla U_{p_{1}}\right\|}=1$, which leads to $\left\|\nabla U_{p_{1}}\right\|=\mathcal{P}$.

Equation (3) can be numerically solved by simple ordinary differential equations techniques like Newton's or Runge-Kutta's. To numerically solve equation (4), classic finite differences schemes tend to be unstable. In [19], Tsitsiklis introduced a new method that was independently reformulated by Sethian in [17]. It 
relies on a one-sided derivative looking in the up-wind direction of the front, and it gives the correct viscosity solution. This algorithm is known as the Fast Marching algorithm and is now widely used. It was used in [10] to solve equation (4) and find globally minimizing contours in images. More details on its background and implementation can be found in [18,9]. It is important to highlight a major advantage of this algorithm. After a simple initialization of $U_{p_{1}}$ over the grid domain, setting $U_{p_{1}}\left(p_{1}\right)=0$ and $U_{p_{1}}(p)=\infty$ for all other points $p$, only one pass over the grid is needed. By using a min-heap data structure, an $O(N \log N)$ complexity can be ensured on a grid of $N$ nodes.

\subsection{Euler-Lagrange and Eikonal equations}

We are now working in a 3D space. The definition of the geodesic active energy related to curves remains the same when considering a curve traced in 3D. Henceforth, $E$ will then denote this curve energy. We are now interested in the relation between the critical curves of energy $E$ (definition given below) and the Eikonal equation solution $U_{p_{1}}$ (viscosity solution).

We use the following usual definitions, the unit tangent vector of curve $C$ at point $C(t)$ is $\vec{T}=C^{\prime}(t) /\left\|C^{\prime}(t)\right\|$. If $s$ is the arclength parameterization of $C$, the curvature $\kappa$ relative to $C$ is defined by $\kappa=\left\|\frac{d^{2} C}{d s^{2}}\right\|$, and its normal vector by $\vec{n}=\frac{1}{\kappa} \frac{d^{2} C}{d s^{2}}$.

The binormal vector of $C$ is defined by $\vec{b}=\vec{T} \wedge \vec{n}$. From these definitions it is easy to see that the derivation of the tangent vector with respect to any parameterization $t$ gives $\vec{T}^{\prime}=\kappa\left\|C^{\prime}\right\| \vec{n}$. Using this definition we can now obtain the Euler-Lagrange equation of the energy $E$ in $3 \mathrm{D}$.

Theorem 1 (Euler-Lagrange of $E$ in 3D) Any curve $C$ traced in $\mathbb{R}^{3}$, closed or having fixed extremities, which is a local minimum of energy $E$ is a solution of

$$
\nabla \mathcal{P}(C) \cdot \vec{n}=\mathcal{P}(C) \kappa \text { and } \nabla \mathcal{P}(C) \cdot \vec{b}=0
$$

Vectors $\vec{n}$ and $\vec{b}$ are the normal and the binormal vectors of $C$ and $\kappa$ its curvature (see [6] for more details on curves in 3D).

Proof:

We are looking for a necessary condition for a curve to be a minimum of the geodesic energy $E$. We consider then an open curve $C$ parameterized on $[0,1]$ and we suppose this curve to be a local minimum of $E$. We compute the first variation of $E$ around $C$.

Let $\gamma$ be a differential curve also parameterized on $[0,1]$, such that $\gamma(0)=\gamma(1)=$ 
0 (we assume the extremities of $C$ are fixed). The first variation of $E$ in the direction of $\gamma$, around $C$ is obtained by $d_{\gamma} E(\mathcal{C})=\lim _{\lambda \rightarrow 0} \frac{E(\mathcal{C}+\lambda \gamma)-E(\mathcal{C})}{\lambda}$. Then, defining the real function $F: \lambda \rightarrow E(\mathcal{C}+\lambda \gamma)$, we have $d_{\gamma} E(\mathcal{C})=F^{\prime}(0)$. We now use the definition of $E=\int \mathcal{P}(\mathcal{C}(s)) d s$ and so we have

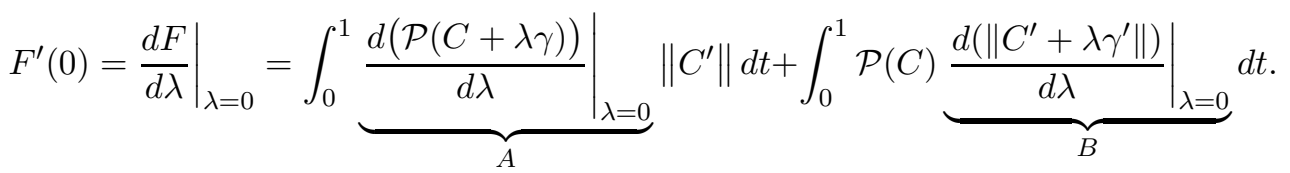

Then, by chain rule derivation we obtain,

$$
A=\nabla \mathcal{P}(C) \cdot \gamma \quad \text { and } \quad B=\frac{C^{\prime}}{\left\|C^{\prime}\right\|} \cdot \gamma^{\prime}=\vec{T} \cdot \gamma^{\prime}
$$

By integration by parts, we modify the form of the second term of (6), note that the values at 0 and 1 of $\gamma$ are null, hence we have

$$
\int_{0}^{1} \mathcal{P}(C) \vec{T} \cdot \gamma^{\prime} d t=-\int_{0}^{1}\left[\left(\nabla \mathcal{P}(C) \cdot C^{\prime}\right) \vec{T}+\mathcal{P}(C) \vec{T}^{\prime}\right] \cdot \gamma d t
$$

At last, by replacing $\vec{T}^{\prime}$ in equation (8), and by using the expression $A$ of equation (7), we obtain the new form of (6): $d_{\gamma} E(C)=\int(\nabla \mathcal{P}(C)-(\nabla \mathcal{P} \cdot \vec{T}) \vec{T}$ $\mathcal{P}(C) \kappa \vec{n}) \cdot \gamma\left\|C^{\prime}\right\| d t$. However, since we suppose that curve $C$ is a local minimum of $E$, the first variation of this energy around this curve is zero, and this is true for all direction $\gamma$. We thus deduce the Euler-Lagrange equation:

$$
\nabla \mathcal{P}(C)-(\nabla \mathcal{P} \cdot \vec{T}) \vec{T}-\mathcal{P}(C) \kappa \vec{n}=0
$$

Let us project this equation onto the Frenet basis $\{\vec{T}, \vec{n}, \vec{b}\}$ so that we get at last equation (5).

Notice that since $C^{\prime}(s)=\vec{T}$ and $C^{\prime \prime}(s)=\kappa \vec{n}$, equation (9) can be rewritten as

$$
\nabla \mathcal{P}(C)=\left(\mathcal{P}(C) C^{\prime}\right)^{\prime}
$$

Strictly speaking, the converse of the Euler-Lagrange condition is false. Not all solutions of this equation are local minima. This is why we give the following definition

Definition 2 (Critical curves) We say that $C$ is a critical curve of the energy $E$ if $C$ is a solution of the Euler-Lagrange equation (5). 
We now study the link between critical curves and the viscosity solution, $U_{\mathbf{p}_{1}}$, of the Eikonal equation. To that end we introduce

Definition 3 (field lines) We will say that $\mathcal{C}$ is a field line of $\nabla U_{p_{1}}$ if it is the solution of the ordinary differential equation

$$
\left\{\begin{array}{l}
\frac{d \mathcal{C}(t)}{d t}=-\nabla U_{p_{1}}(\mathcal{C}(t)) \\
\mathcal{C}(0)=\mathbf{p} .
\end{array}\right.
$$

where $\mathbf{p}$ is a point of the image domain.

And we have the following property:

Theorem 4 (Field Lines and Euler-Lagrange equation) If $U_{\mathrm{p}_{1}}$ is solution to the problem $\left\|\nabla U_{\mathbf{p}_{1}}\right\|=\mathcal{P}$ with $U_{\mathbf{p}_{1}}\left(\mathbf{p}_{1}\right)=0$, every line field of $\nabla U_{\mathbf{p}_{1}}$ is a critical curve of the geodesic energy $E$.

Proof:

Let $\mathbf{p}$ be a point in the image domain such that, in $\mathbf{p}$, the viscosity solution of the Eikonal equation $U_{\mathbf{p}_{1}}$ is of class $C^{2}$ ( $\mathbf{p}$ is such that equation (11) has only one solution). We can then compute the derivative of the two members of the squared Eikonal equation $\left\|\nabla U_{\mathbf{p}_{1}}\right\|^{2}=\mathcal{P}^{2}$ at point $\mathbf{p}$, which drives us to

$$
\nabla^{2} U_{\mathbf{p}_{1}}(\mathbf{p}) \nabla U_{\mathbf{p}_{1}}(\mathbf{p})=\mathcal{P} \nabla \mathcal{P}
$$

Consider now a field line of $\nabla U_{\mathbf{p}_{1}}$. After a reparameterization $C_{1}(s)=C(L-$ $s$ ) in order to change the sign of the first equality of (11), if $s$ is the arclength parameterization of $C_{1}$, we can write:

$$
\frac{d C_{1}(s)}{d s}=\frac{-C^{\prime}(t)}{\left\|C^{\prime}(t)\right\|}=\frac{\nabla U_{\mathbf{p}_{1}}(C(t))}{\left\|\nabla U_{\mathbf{p}_{1}}(C(t))\right\|}=\frac{\nabla U_{\mathbf{p}_{1}}\left(C_{1}(s)\right)}{\left\|\nabla U_{\mathbf{p}_{1}}\left(C_{1}(s)\right)\right\|}=\frac{\nabla U_{\mathbf{p}_{1}}\left(C_{1}(s)\right)}{\mathcal{P}\left(C_{1}(s)\right)} .
$$

since $U_{\mathbf{p}_{1}}$ satisfies the Eikonal equation. We thus have $\mathcal{P}\left(C_{1}(s)\right) \frac{d C_{1}(s)}{d s}=\nabla U_{\mathbf{p}_{1}}\left(C_{1}(s)\right)$. The computation of the derivative of this last equation with respect to $s$ gives $\frac{d}{d s}\left[\mathcal{P}\left(C_{1}(s)\right) \frac{d C_{1}(s)}{d s}\right]=\nabla^{2} U_{\mathbf{p}_{1}}\left(C_{1}(s)\right) \frac{d C_{1}(s)}{d s}=\frac{\nabla^{2} U_{\mathbf{p}_{1}}\left(C_{1}(s)\right) \nabla U_{\mathbf{p}_{1}}\left(C_{1}(s)\right)}{\mathcal{P}\left(C_{1}(s)\right)}=\nabla \mathcal{P}\left(C_{1}(s)\right)$ using equation (12), and this is exactly the Euler Lagrange equation of energy $E$ as written in (10).

Note that Kimmel et al. presented a similar proof in the two dimensional case in [13]. Our proof is valid for any dimension (in particular in 3D). On the other hand, we know that the action map $U_{\mathbf{p}_{1}}$ is the unique viscosity solution bounded from below of the Eikonal equation (see [14]). In the present case, where the 
boundary condition is only $U_{\mathbf{p}_{1}}\left(\mathbf{p}_{1}\right)=0$, the regularity of $U_{\mathbf{p}_{1}}$ only depends on the regularity of the potential function $\mathcal{P}$. In this paper we do not consider the problem concerning points where the action map is not regular enough for its gradient to be defined. In practice, numerical approximations can solve the problem when the only goal is to compute a minimal path between two points. As a matter of fact, one of the main interests of theorems 1 and 4 is that they allow us to consider efficient numerical approaches in order to compute minimal paths to point $\mathbf{p}_{1}$ from any point in the image domain. An extension to 3D of Fast Marching and minimal paths is straightforward. The authors of [11] used it to find centerlines in 3D tubular structures. After the computation of the action map by this extension, the minimal path is obtained by gradient descent, solving equation (3), like in the $2 \mathrm{D}$ case. We now present our first extension of minimal paths to surface segmentation.

\subsection{Minimal paths between two curves}

We now seek to extract a surface that contains two constraining curves $\Gamma_{1}$ and $\Gamma_{2}$ using the method of minimal paths outlined in the previous section, that is we look for an extension to 3D surface of the minimal path segmentation. Intuitively, we see that, if potential $\mathcal{P}$ is correctly tailored, every minimal path between a point $\mathbf{p}_{1} \in \Gamma_{1}$ and a point $\mathbf{p}_{2} \in \Gamma_{2}$ should belong (or at least be close enough) to the surface we wish to extract. A naive approach for the generation of a 'surface' is to build minimal paths between all the points of $\Gamma_{1}$ and $\Gamma_{2}$ (Note that the Hopf-Rinow theorem (see section 5-3 of [6]) ensures the existence of a minimal path between any two points in the metric space induced by potential $\mathcal{P}$ ). Hence, each point of $\Gamma_{1}$ would be associated to every point of $\Gamma_{2}$. Clearly, from a computational point of view, this would be expensive (at least $n$ action maps to build and $n \times n$ gradient descents, if $n$ is the number of points of the discretized versions of $\Gamma_{1}$ and $\Gamma_{2}$ ), and many of these numerous associations would not be relevant. We thus propose to exploit the set of minimal paths between each point of curve $\Gamma_{2}$ and curve $\Gamma_{1}$ considered as a whole. We then have to work with paths between points and curves.

Definition 5 (Path between a point and a curve) We call path between a point $\mathbf{p}$ and a curve $\Gamma$, a curve $\gamma$ such that $\gamma(0)=\mathbf{p}$ and $\gamma(1) \in \Gamma(\gamma$ is parameterized in $[0,1])$.

Expecting a result similar to theorem 4, it is natural to introduce an action map with respect to one of the curves given by the user.

Definition 6 (Curve's Minimal action map) We call minimal action map with respect to curve $\Gamma$ and potential $\mathcal{P}$, the function $U_{\Gamma}$ with real values, that associates 
to any point $\mathbf{p}$ of the image domain, the value

$$
U_{\Gamma}(\mathbf{p})=\min _{\substack{\gamma \text { between } \\ \mathbf{p} \text { and } \Gamma}}\{E(\gamma)\}=\min _{\substack{\gamma \text { between } \\ \mathbf{p} \text { and } \Gamma}}\left\{\int_{0}^{1} \mathcal{P}(\gamma(t))\left\|\gamma^{\prime}(t)\right\| d t\right\} .
$$

Notice that the minimal path between $\mathbf{p}$ and $\Gamma$ is the path having the minimal geodesic energy among all curves between a point $\mathbf{q} \in \Gamma$ and $\mathbf{p}$. Function $U_{\Gamma}$ is a distance function from $\Gamma$ in the Riemannian space induced by $\mathcal{P}$ and thus $U_{\Gamma}=\min _{\mathbf{q} \in \Gamma}\left\{U_{\mathbf{q}}\right\}$. This equality anticipates the following result, for which a rigorous proof can be found in the work of Mantegazza and Mennucci in [14].

Theorem 7 The action map $U_{\Gamma}$ is the only viscosity solution bounded from below of the Eikonal equation

$$
\left\|\nabla U_{\Gamma}\right\|=\mathcal{P}, \text { and } \forall p \in \Gamma, U_{\Gamma}(p)=0 .
$$

Notice that if there exists only one point $\mathbf{q}_{0}$ of $\Gamma$ such that $U_{\Gamma}(\mathbf{p})=\min _{\mathbf{q} \in \Gamma}\left\{U_{\mathbf{q}}(\mathbf{p})\right\}=$ $U_{\mathbf{q}_{0}}(\mathbf{p})$, then we have $\left\|\nabla U_{\Gamma}(\mathbf{p})\right\|=\left\|\nabla U_{\mathbf{q}_{0}}(\mathbf{p})\right\|=\mathcal{P}(\mathbf{p})$. The difficulty is that the uniqueness of point $\mathbf{q}_{0}$ is not always satisfied.

Again, a major interest of this last property, is the fact that the Fast Marching algorithm can also be used to rapidly compute a numerical approximation of $U_{\Gamma}$, the only difference is that we have a different boundary condition. The following property will then provide us a tool for rapidly computing minimal paths between a point and a curve.

Theorem 8 (Field Lines of $U_{\Gamma}$ ) If $C$ is a field line of $\nabla U_{\Gamma}$, containing point $\mathbf{p}$, then $C$ is a critical curve of the geodesic energy $E$ between point $\mathbf{p}$ and curve $\Gamma$.

Concerning this property, mathematically speaking, we are not ensured that $U_{\Gamma}$ is regular enough to define its gradient. As Mennucci shows in [15], the regularity of $U_{\Gamma}$ depends upon the regularity of $\mathcal{P}$ but also on the regularity of $\Gamma$. Notice also that whatever the shape of curve $\Gamma$ is, even if the potential $\mathcal{P}$ is $C^{\infty}$, there always exist points where $U_{\Gamma}$ is not $C^{1}$. The interesting fact is that the set of these points has a zero $\mathcal{H}^{3}$ - measure (Hausdorff measure of third degree), so that in practice, ambiguities in the determination of the gradient can easily be solved.

\subsection{Minimal path set between two curves $\Gamma_{1}$ and $\Gamma_{2}$}

Let us now define our minimal path set. Curves $\Gamma_{1}$ and $\Gamma_{2}$ are exploited as the initialization of the model and as incorporated user information. The approach is 
based on considering a network of paths that globally minimizes an energy associated to the image. This network is used to generate a surface that contains the constraining curves and provides a segmentation of the object lying between them. A curve $\gamma_{\Gamma_{1}}^{q}$ is a path between a point $q$ and curve $\Gamma_{1}$ if $\gamma_{\Gamma_{1}}^{q}(0)=q$ and $\gamma_{\Gamma_{1}}^{q}(L) \in$ $\Gamma_{1}$. A path network $\mathcal{N}_{\Gamma_{1}}^{\Gamma_{2}}$, between the points of $\Gamma_{2}$ and curve $\Gamma_{1}$, is the set $\mathcal{N}_{\Gamma_{1}}^{\Gamma_{2}}=\left\{\gamma_{\Gamma_{1}}^{q}\right\}_{q \in \Gamma_{2}}$, where it is supposed that every point $q$ of $\Gamma_{2}$ is visited only once. Globally minimal path between the points of $\Gamma_{2}$ and curve $\Gamma_{1}$ are easily found. Using theorem 8 , a minimal path between $\Gamma_{1}$ and a point $q$ (further noted $\mathcal{C}_{\Gamma_{1}}^{q}$ ), with respect to the energy $E=\int_{0}^{L} \mathcal{P}(\mathcal{C}(s)) d s$, is found as a field line of $\nabla U_{\Gamma_{1}}$ :

$$
\frac{d \mathcal{C}_{\Gamma_{1}}^{q}}{d t}(t)=-\nabla U_{\Gamma_{1}}\left(\mathcal{C}_{\Gamma_{1}}^{q}(t)\right) \text { with } \mathcal{C}_{\Gamma_{1}}^{q}(0)=q
$$

By solving equation (16), using each point of $\Gamma_{2}$ as part of the initial condition $\left(\mathcal{C}_{\Gamma_{1}}^{q}(0)=q\right)$, we globally minimize the energy, producing a minimal energy network:

Definition 9 We call minimal path set between $\Gamma_{1}$ and the set of points of $\Gamma_{2}$, with respect to potential $\mathcal{P}$, the set

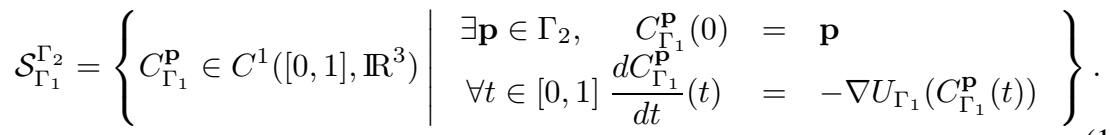

$U_{\Gamma_{1}}$ being the solution to the Eikonal equation $\left\|\nabla U_{\Gamma_{1}}\right\|=\mathcal{P}, \forall \mathbf{p} \in \Gamma_{1}, U_{\Gamma_{1}}(\mathbf{p})=$ 0 .

The minimal network is thus the set of all solutions of the ordinary differential equation (16) when varying its initial condition along $\Gamma_{2}$. Up to a reparameterization, assume every minimal path (respectively curve $\Gamma_{2}$ ) is parameterized on an interval $J$ (respectively $I$ ). $\mathcal{S}_{\Gamma_{1}}^{\Gamma_{2}}$ can then be considered as a mapping (since minimal paths cannot cross without merging) from $I \times J$ to $\Omega$, such that for all pair $(u, v) \in I \times J, \mathcal{S}_{\Gamma_{1}}^{\Gamma_{2}}(u, v)=\mathcal{C}_{\Gamma_{1}}^{\Gamma_{2}(u)}(v)$. Using this map for segmentation follows the same intuition as in [3], where the hypothesis is made that each path of $\mathcal{S}_{\Gamma_{1}}^{\Gamma_{2}}$ is within a small distance from the surface to extract.

If potential $\mathcal{P}$ is correctly chosen, the set of curves $\mathcal{S}_{\Gamma_{1}}^{\Gamma_{2}}$ should be near the surface that we wish to extract. In order to illustrate this fact, consider the synthetic example given in figure 2. There we show some curves belonging to $\mathcal{S}_{\Gamma_{1}}^{\Gamma_{2}}$ when the potential is obtained from a binary synthetic image of a vase.

Unfortunately, as can be understood from [14], and illustrated in figure 6middle, in the general case the map $\mathcal{S}_{\Gamma_{1}}^{\Gamma_{2}}(\cdot, \cdot)$ lacks the fundamental property of 


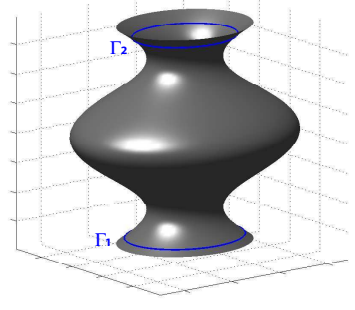

(a)

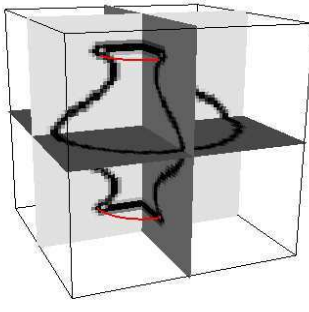

(b)

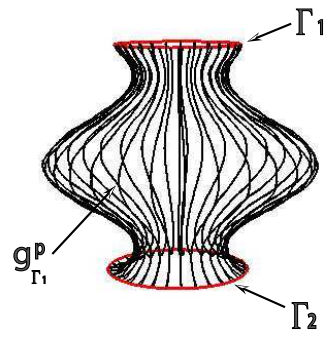

$(c)$

Figure 2: (a) represents the original surface from which we generated the synthetic image. In (b) we show three orthogonal slices of the 3D potential obtained by filtering the binary image and taking a decreasing function of its gradient norm. (c) Shows some minimal paths computed between the two user given curves.

continuity. For that reason, it is insufficient for segmentation. In order to cope with this difficulty, two different solution were proposed in [3]. However, they still have some constraints and therefore we introduce in the next section a novel approach for the generation of a surface using the minimal path network. This surface shall be defined as the zero level set of a function $\Psi$ which solves a certain transport equation.

\section{Implicit defi nition of a surface containing the minimal path set}

In order to simplify our description, $\Gamma_{1}$ and $\Gamma_{2}$ are assumed to be two non-intersecting planar, closed curves. We look for a real function $\Psi$, defined on the image domain $\Omega$, such that $\mathcal{S}_{\Gamma_{1}}^{\Gamma_{2}}$ is contained in its zero level set (further noted $\Psi^{-1}(\{0\})$ ). Having no a priori knowledge on the properties $\Psi$ should satisfy, we shall suppose that $\Psi$ is continuously differentiable and we first look for a necessary condition based on our knowledge of the minimal path network. Further, this condition is exploited to formulate a sufficient condition and finally give a consistent description of function $\Psi$. 


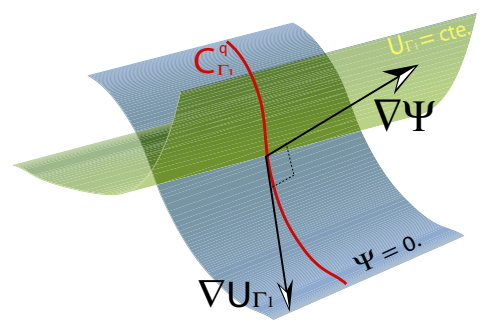

Figure 3: Along a minimal path the gradient of $\Psi$ should be perpendicular to the gradient of the action map.

\subsection{Searching for an implicit function}

As in section 2.4 , we denote by $\mathcal{C}_{\Gamma_{1}}^{q}$ a minimal path from a point $q \in \Omega$ to curve $\Gamma_{1}$, and we suppose that $J$ is its parameterization interval. The minimal path set $\mathcal{S}_{\Gamma_{1}}^{\Gamma_{2}}$ can also be considered as a subset of $\Omega, p \in \mathcal{S}_{\Gamma_{1}}^{\Gamma_{2}}$ means that $p$ is a point belonging to a minimal path (joining a point of $\Gamma_{2}$ and $\Gamma_{1}$ ). Let us first assume that $\Psi$ is a continuously differentiable function, defined on $\Omega$ such that $\mathcal{S}_{\Gamma_{1}}^{\Gamma_{2}} \subset \Psi^{-1}(\{0\})$. This means that for all minimal path $\mathcal{C}_{\Gamma_{1}}^{q}$ we have $\forall s \in$ $J, \Psi\left(\mathcal{C}_{\Gamma_{1}}^{q}(s)\right)=0$. From the derivative with respect to $s$ of this relation we obtain $\forall s \in J, \nabla \Psi\left(\mathcal{C}_{\Gamma_{1}}^{q}(s)\right) \cdot \frac{d \mathcal{C}_{\Gamma_{1}}^{q}}{d s}(s)=0$. Using relation (16) we deduce the following proposition:

Theorem 10 (Necessary condition) For any real $C^{1}$ function $\Psi$, defined on $\Omega$, and such that $\mathcal{S}_{\Gamma_{1}}^{\Gamma_{2}} \subset \Psi^{-1}(\{0\})$, we have for every point $p$ of $\mathcal{S}_{\Gamma_{1}}^{\Gamma_{2}}$ :

$$
\nabla \Psi(p) \cdot \nabla U_{\Gamma_{1}}(p)=0 .
$$

The perpendicularity of the two gradient vector fields is only necessary on the points of the minimal path network. Hardening this condition and demanding that $\Psi$ satisfies a relation similar to (18) everywhere in $\Omega$, should lead to a sufficient relation for the minimal paths to be contained in $\Psi^{-1}(\{0\})$.

It is interesting to note that we only want to act on the zero level set of $\Psi$; this gives the opportunity to introduce a regularization term in the previous equation. For this purpose we introduce a regular (at least continuously differentiable) real function $G$ that satisfies $G(0)=0$. We have the following proposition :

Theorem 11 (Sufficient condition) If $\Psi$ is $C^{1}$ satisfying :

$$
\left\{\begin{array}{rr}
\left(C_{1}\right) & \forall p \in \Omega, \nabla \Psi(p) \cdot \nabla U_{\Gamma_{1}}(p)+G \circ \Psi(p)=0 \\
\left(C_{2}\right) & \forall q \in \Gamma_{2}, \Psi(q)=0
\end{array}\right.
$$


then the minimal path network $\mathcal{S}_{\Gamma_{1}}^{\Gamma_{2}}$ is contained in the zero level set of $\Psi$.

Proof: For every point $q \in \Gamma_{2}$, the values taken by function $\Psi$ along the minimal path $C_{\Gamma_{1}}^{q}$ are given by function $f_{q}=\Psi \circ \mathcal{C}_{\Gamma_{1}}^{q}$. We have, for all $s \in J: \frac{d f_{q}}{d s}(s)=$ $\nabla \Psi\left(C_{\Gamma_{1}}^{q}(s)\right) \cdot \frac{d \mathcal{C}_{\Gamma_{1}}^{q}}{d s}(s) \underbrace{=}_{\text {from }(16)}-\nabla \Psi\left(\mathcal{C}_{\Gamma_{1}}^{q}(s)\right) \cdot \nabla U_{\Gamma_{1}}\left(\mathcal{C}_{\Gamma_{1}}^{q}(s)\right)=G\left(f_{q}(s)\right)$. Thus, the function $f_{q}$ satisfies over the interval $J$ the ordinary differential equation

$$
\frac{d f_{q}}{d s}=G\left(f_{q}\right) .
$$

Furthermore, recall that $C_{\Gamma_{1}}^{q}(0)=q$ and $q \in \Gamma_{2}$. Condition $\left(C_{2}\right)$ establishes then that $f_{q}(0)=0$. Consequently, since $G$ is a differentiable function such that $G(0)=0$, the unique solution to (20) is $f_{q}=0$ on $J$. A simple proof of this can be obtained by denoting $H(u)=\frac{G(u)}{u}$ and $H(0)=G^{\prime}(0)$. Since $G$ is continuously differentiable and $G(0)=0, H$ is well defined and continous. Denoting $H_{1}$ such that $H_{1}^{\prime}(u)=G(u)$ and multiplying equation 20 by $\exp \left(-H_{1}\right)$, we get that the derivative of $\exp \left(-H_{1}\right) f_{q}$ is equal to zero. the latter function is thus constant, and since its value at 0 is zero, we have $f_{q}=0$ on $J$. In other words, the unique value taken by function $\Psi$ along any minimal path originated on a point $q$ of $\Gamma_{2}$ is zero, which is exactly $\mathcal{S}_{\Gamma_{1}}^{\Gamma_{2}} \subset \Psi^{-1}(\{0\})$.

A too quick examination of the conditions given in (19) may not be enough to understand why this implicit approach will produce a better segmentation method than explicitly generating a finite number of paths of $\mathcal{S}_{\Gamma_{1}}^{\Gamma_{2}}$ followed by interpolation (as in [3]). The following proposition makes this clear by establishing that the zero level set of the solution to (19) has a very particular structure: it is completely composed of globally minimal paths.

Theorem $12\left(\Psi^{-1}(\{0\})\right.$ structure) If $\Psi$ satisfies the same conditions as in theorem 11, then for all $p \in \Psi^{-1}(\{0\})$, the minimal path $\mathcal{C}_{\Gamma_{1}}^{p}$ joining $p$ to $\Gamma_{1}$ is also included in this zero level set.

Proof: If $\Psi$ satisfies the conditions of theorem 11, then for any point $p$ of $\Omega$, function $f_{p}=\Psi \circ C_{\Gamma_{1}}^{p}$ satisfies the ordinary differential equation (20). Thus the values of $\Psi$ along minimal path $C_{\Gamma_{1}}^{p}$ only depend on the initial value $f_{p}(0)=\Psi(p)$. If $p$ is on the zero level set of $\Psi$ then $f_{p}(0)=\Psi(p)=0$ and, as in the proof of the previous proposition, $\Psi \circ C_{\Gamma_{1}}^{p}=0$. This means that every point of $\Psi^{-1}(\{0\})$ belongs to a minimal path.

Being minimal with respect to the geodesic energy $E$, these paths tend to be traced on the object to extract (as $\Gamma_{1}$ and $\Gamma_{2}$ ). This explains the better results, 


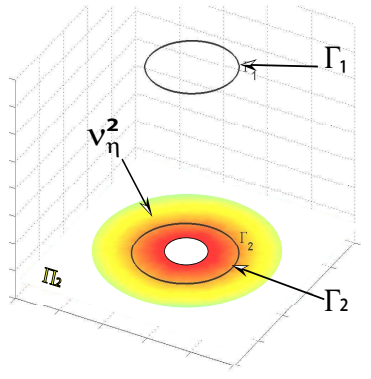

Figure 4: Boundary conditions for the transport problem $\left(\Pi_{1}\right.$ and $\Pi_{2}$ are not necessary parallel).

compared to [3], obtained with our method. A good example is given in figure 6, which demonstrates, on a synthetic image, how this approach gives good results where clearly $\mathcal{S}_{\Gamma_{1}}^{\Gamma_{2}}$ is insufficient for segmentation.

In the next sections we explicitly give the problem to be solved in order to build the segmentation of an object from the two constraining curves. Most likely traced by a human user (or a $2 \mathrm{D}$ segmentation process), $\Gamma_{1}$ and $\Gamma_{2}$ will be supposed to be planar curves.

\subsection{Using minimal paths for surface segmentation}

We further denote by $\Pi_{1}, \Pi_{2}$ the intersection of the planes containing $\Gamma_{1}$ and $\Gamma_{2}$ with the image domain (remember that this is purely a practical condition, it is not necessary that these curves are planar but, if not, the description of the boundaries of our problem is more complicated). The functions $d_{1}, d_{2}$ are the signed distance functions to these curves, positive in their interior and defined on $\Pi_{1}$ and $\Pi_{2}$ respectively. Notice that at each point $q \in \Gamma_{2}, d_{2}(q)=0$.

Consider now the closed set $\mathcal{V}_{\eta}^{2}=\left\{p \in \Pi_{2}\right.$ such that $\left.\left|d_{2}(p)\right| \leq \eta\right\}$, where $\eta$ is a real positive value (see figure 4 ). Inspired by theorem 11 , we consider the open set $\mathcal{O}=\operatorname{int}(\Omega)-\mathcal{V}_{\eta}^{2}$, where $\operatorname{int}(\Omega)$ is the interior of the image domain, and search for $\Psi$ as the solution to the Cauchy problem defined on $\Omega$ :

$$
\begin{cases}\nabla \Psi(p) \cdot \nabla U_{\Gamma_{1}}(p)+G \circ \Psi(p)=0 & \text { if } p \in \mathcal{O}, \\ \Psi(p)=d_{2}(p) & \text { if } p \in \mathcal{V}_{\eta}^{2}, \\ \Psi(p)=\min _{p \in \Pi_{2}}\left(d_{2}(p)\right) & \text { if } p \in \delta \Omega .\end{cases}
$$

$\delta \Omega$ is the boundary of the image domain $\Omega$. Notice that outside $\Gamma_{2}$, the signed distance $d_{2}$ is negative, and thus the minimum value taken in the third equation of (21) is the largest distance to $\Gamma_{2}$ in $\Pi_{2}$ with a minus sign. This is a stationary transport 
problem where the function $G$ stands for the source term. It is beyond the scope of this paper to present the theoretical details of the existence and uniqueness. As a matter of fact, numerical approaches (see section 3.4) that take in consideration the presence of possible discontinuities of the function $\Psi$ were proposed before a theoretical framework was established. Let us now observe the influence on our problem of the choice of the function $G$.

\subsection{Choice of the function $G$}

As presented in the previous section, the function $G$ is only required to be continuously differentiable and satisfy $G(0)=0$. It is interesting to examine some possible choices of $G$ and to see its influence on the solution $\Psi$.

$\mathbf{G}=\mathbf{0}$ : With this choice, problem (21) becomes a stationary transport problem. Equation (20) is in this case $\frac{d f_{q}}{d s}=0$, so that for every point $p$ of $\Omega$ function $\Psi \circ C_{\Gamma_{1}}^{p}$ is constant ( $\Psi$ is constant along any minimal path). $\Psi$ 'transports' the values of the boundary $\mathcal{V}_{\eta}^{2} \cup \delta \Omega$ along the minimal paths (the transport problem has been studied from a theoretical point of view see for example [1] and references within, results of existence and uniqueness have been given by Bouchut et. al. in [4] and L. Ambrosio in [1]).

Even though this choice seems the most natural, the resulting $\Psi$ may present discontinuities. By construction, every curve solution of problem (16) (a minimal path) will join curve $\Gamma_{1}$. If $p$ and $q$ are two points of $\mathcal{V}_{\eta}^{2}$ such that $d_{2}(p) \neq d_{2}(q)$ then along each of the paths $C_{\Gamma_{1}}^{p}$ and $C_{\Gamma_{1}}^{q}, \Psi$ will take these two different values. This supposes that the different values transported by $\Psi$ from the boundaries will 'collapse' on curve $\Gamma_{1}$ producing discontinuities.

In figure 5(a) we show a synthetic example where the surface to extract is a cylinder-like object having a spherical protuberance, the two constraining curve being traced on parallel planes, normal to the cylinder's axis. We show some level sets of function $\Psi$ (obtained using the numerical method presented in section 3.4) on two orthogonal planes, these level sets collapse within a short distance of curve $\Gamma_{2}$. This phenomenon is similar to the merging of minimal paths that caused interpolation problems in [3].

$\mathbf{G} \circ \mathbf{\Psi}=\alpha \mathbf{\Psi}: \alpha$ is supposed to be a positive constant. With this choice, equation (20) becomes $\frac{d f_{q}}{d s}=-\alpha f_{q}$, whose solution is $f_{q}(s)=\Psi(q) \exp (-\alpha s)$. Along minimal paths, the function $\Psi$ is no longer constant, its value decreases in a exponential manner. With this simple approach it is possible to avoid the collapsing effect of the previous case, and have level sets of $\Psi$ that are more regularly spaced. This can be seen as a way to regularize our problem without the necessity of adding smoothing term based on higher degree derivatives. In our implementation, $\alpha$ is still a parameter but automatic approaches to find this constant are being studied. 
In figure 5(b) we show the effect of this regularization on the same synthetic image as in figure 5(a).

\subsection{Implementation Issues}

We now describe an efficient algorithm for the numerical implementation of the transport problem (21). Unlike [3], minimal paths are not to be computed directly in this implicit approach. We only numerically calculate solutions to the Eikonal and stationary transport equations.

To numerically solve the Eikonal equation (15) classic finite difference schemes tend to be unstable. Generally it is preferable to use consistent algorithms using upwind differences (derivative approximations are chosen looking in the direction from which the information is flowing) as fast marching [17].

The stationary transport equation, as with most first order partial differential equations whose characteristics intersect, is difficult to solve numerically. In fact, in the general case ( $\mathcal{P}$ is supposed to be a bounded and continuous function), there is no classical solution defined in all $\Omega$, and the weak solution $\Psi$ can present discontinuities. Many implementations of the transport equation in its non-static expression have been proposed in the modeling of geophysical phenomena. Here we will concentrate on a first order, fast algorithm which is less constrained since only the zero level set of the solution matters in our approach.

In order to simplify notation, the symbol $V$ shall be used to refer to the gradient $\nabla U_{\Gamma_{1}}$. One of the first numerical approaches for solving the transport equation proposes a first order approximation of the gradient $\nabla \Psi$ that follows the direction in which information propagates. This discretization is the upwind approach and consists in choosing the approximation of $\frac{\partial \Psi}{\partial \delta}$ following the sign of the components $V_{\delta}$ (where $\delta=x, y$ ou $z$ ) of $V$. Recently, A. Yezzi and J. L. Prince used this scheme in [20] for the numerical solution of equation $\nabla \Psi \cdot T=1$ (where $T$ was a known vector field). At last, although this scheme is of relatively low precision and dissipative, it gives satisfactory results in our experiments with an acceptable convergence speed.

If $\Psi^{i, j, k}$ is the value of the numerical approximation of $\Psi$ at point $[i ; j ; k]$ of the discrete square grid, we shall denote the left and right approximations of the partial derivatives by:

$$
D^{-x} \Psi=\frac{\Psi^{i, j, k}-\Psi^{i-1, j, k}}{h}, D^{+x} \Psi=\frac{\Psi^{i+1, j, k}-\Psi^{i, j, k}}{h}
$$

(similarly in the $y$ and $z$ directions) where $h$ is the discretization step, identical in all three spatial directions. Our scheme for solving the stationary transport problem 

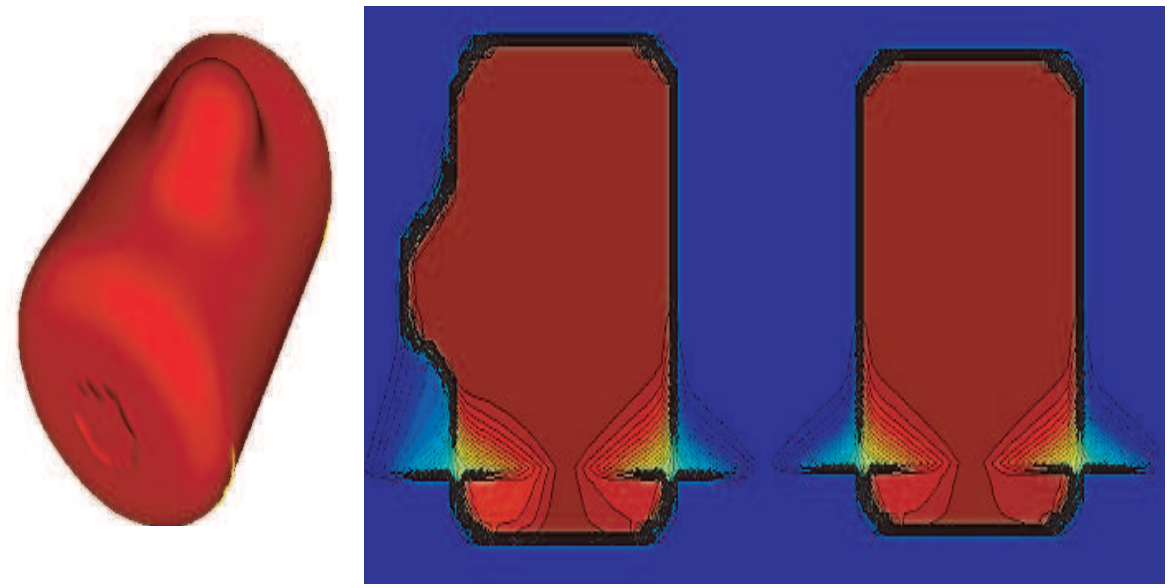

(a) Result obtained when solving the transport equation without second term.

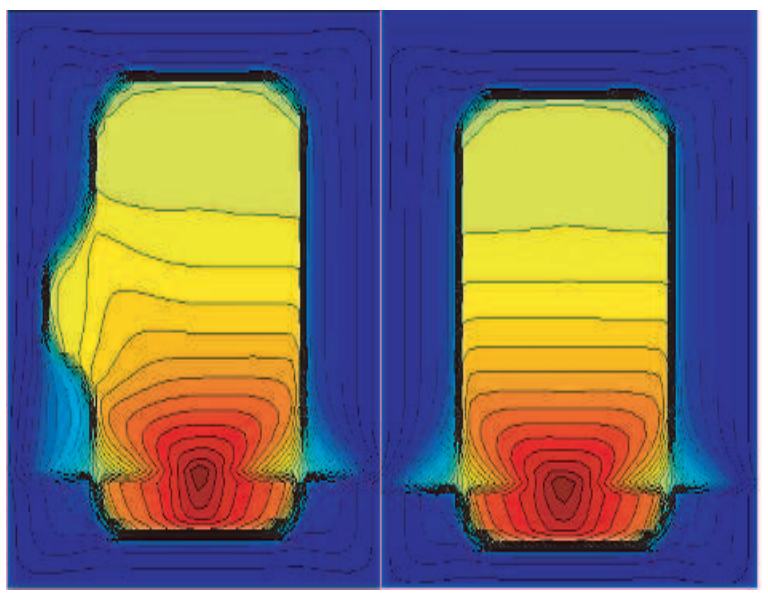

(b) Result obtained when using a second term of the form $G(\Psi)=\alpha \Psi$

Figure 5: Intersection of two planes with the level sets of the numerical solution of our transport equation when the potential used is $\mathcal{P}=\left(1+|\nabla I|^{2}\right)^{-1}$, and $I$ is the binary image obtained from the synthetic volume shown in (a). 
$V \cdot \nabla \Psi=-\alpha \Psi$ is then

$$
\begin{aligned}
V_{x}^{i, j, k}\left(D^{-x} \Psi^{i, j, k} \text { or } D^{+x} \Psi^{i, j, k}\right)+V_{y}^{i, j, k}\left(D^{-y} \Psi^{i, j, k} \text { or } D^{+y} \Psi^{i, j, k}\right) & + \\
V_{z}^{i, j, k}\left(D^{-z} \Psi^{i, j, k} \text { or } D^{+z} \Psi^{i, j, k}\right) & =-\alpha \Psi^{i, j, k},
\end{aligned}
$$

where the value of the vector $V$ at grid point $(i, j, k)$ is $\left(V_{x}^{i, j, k}, V_{y}^{i, j, k}, V_{z}^{i, j, k}\right)$. In our problem, the direction in which information propagates is given by the vector $-V$. Therefore, denoting by $H$ the heaviside function defined by $H(x)=$ $\left\{\begin{array}{l}1, \text { if } x \geq 0 \\ 0, \text { else. }\end{array}\right.$, the upwind approximation is:

$$
\begin{aligned}
& V_{x}^{i, j, k}\left(D^{-x} \Psi^{i, j, k} H\left(-V_{x}^{i, j, k}\right)+D^{+x} \Psi^{i, j, k} H\left(V_{x}^{i, j, k}\right)\right)+ \\
& V_{y}^{i, j, k}\left(D^{-y} \Psi^{i, j, k} H\left(-V_{y}^{i, j, k}\right)+D^{+y} \Psi^{i, j, k} H\left(V_{y}^{i, j, k}\right)\right)+ \\
& V_{z}^{i, j, k}\left(D^{-z} \Psi^{i, j, k} H\left(-V_{z}^{i, j, k}\right)+D^{+z} \Psi^{i, j, k} H\left(V_{z}^{i, j, k}\right)\right)=-\alpha \Psi^{i, j, k} .
\end{aligned}
$$

Then, denoting $I=(i+1)$ if $V_{x}>0, i-1$ otherwise, and similarly for $J$ and $K$, we have

$$
\begin{gathered}
\left|V_{x}^{i, j, k}\right|\left[\Psi^{I, j, k}-\Psi^{i, j, k}\right]+\left|V_{y}^{i, j, k}\right|\left[\Psi^{i, J, k}-\Psi^{i, j, k}\right] \\
+\left|V_{z}^{i, j, k}\right|\left[\Psi^{i, j, K}-\Psi^{i, j, k}\right]=-\alpha \Psi^{i, j, k}
\end{gathered}
$$

which, by grouping terms with $\Psi^{i, j, k}$, finally leads to the update expression of our algorithm:

$$
\Psi^{i, j, k}=\frac{\left|V_{x}^{i, j, k}\right| \Psi^{I, j, k}+\left|V_{y}^{i, j, k}\right| \Psi^{i, J, k}+\left|V_{z}^{i, j, k}\right| \Psi^{i, j, K}}{\left|V_{x}^{i, j, k}\right|+\left|V_{y}^{i, j, k}\right|+\left|V_{z}^{i, j, k}\right|-\alpha} .
$$

Thus $\Psi^{i, j, k}$ is an expression of three of its neighbor grid points. $V$ is assumed to be known everywhere from the first step computing the minimal action to one curve or to a set of curves. The $\Psi^{i, j, k}$ are known at the beginning on the boundary grid points. We can use a classical sweeping algorithm with this formula.

These equalities can also be exploited, as presented in [20], in a fast marching type scheme that achieves a first order approximation of the solution to our problem in only one grid pass and with a $N \log N$ complexity. When only two of these neighbors are known, we use a reduced formula. For example, when the third one is missing:

$$
\Psi^{i, j, k}=\frac{\left|V_{x}^{i, j, k}\right| \Psi^{I, j, k}+\left|V_{y}^{i, j, k}\right| \Psi^{i, J, k}}{\left|V_{x}^{i, j, k}\right|+\left|V_{y}^{i, j, k}\right|-\alpha} .
$$

In the same way, when only one neighbor is known, for example the first one, we use the formula:

$$
\Psi^{i, j, k}=\frac{\left|V_{x}^{i, j, k}\right| \Psi^{I, j, k}}{\left|V_{x}^{i, j, k}\right|-\alpha}
$$


In the end, our algorithm consists of solving the Eikonal equation first, then the transport equation by means of the same implementation. We thus can achieve very rapid computing times. In the next section we give some results.
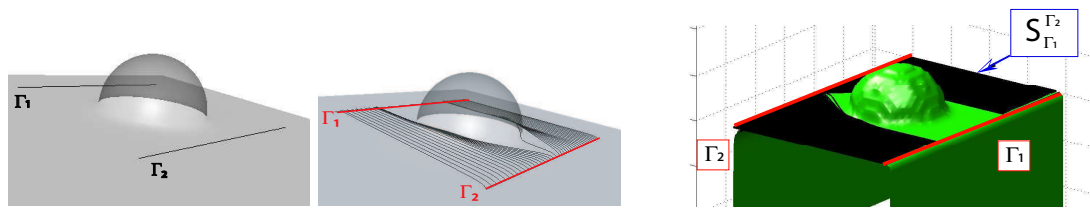

Figure 6: Data is a half-sphere blended with a plane (transparent visualization) and $\Gamma_{1}$ and $\Gamma_{2}$ (black segments). Middle shows some minimal paths of $\mathcal{S}_{\Gamma_{1}}^{\Gamma_{2}}$ taking a short cut around the sphere. On the right, superposition of $\Psi^{-1}(0)$ and set $\mathcal{S}_{\Gamma_{1}}^{\Gamma_{2}}$.

\section{Applications}

We apply our method to some synthetic and real 3D images. In all our examples we used a potential defined for $p$ in the image domain as: $\mathcal{P}(p)=\rho h_{1}\left(\left|\nabla I_{\sigma}(p)\right|\right)+$ $(1-\rho) h_{2}\left(\Delta I_{\sigma}(p)\right)$, where $h_{1}$ and $h_{2}$ are two functions bounded between 0 and 1 and where $I_{\sigma}$ is the convolution of the given image with a Gaussian kernel of variance $\sigma$. Typically, $h_{1}(x)=\frac{1}{1+x^{2} / \lambda^{2}}$, where $\lambda$ is a user defined contrast factor that can be computed as an average gradient value, and $h_{2}$ is chosen to be a zero crossing detector.

Figure 6 represents a sphere blended with a plane. The set of minimal paths $\mathcal{S}_{\Gamma_{1}}^{\Gamma_{2}}$ is unable to provide enough information for the extraction of the surface, since no minimal path 'climbs' on the sphere surface. Nonetheless, the zero level set of the corresponding $\Psi$ function reconstructs perfectly the surface. Our implicit method recovers more information than the minimal paths and we obtain the complete surface.

In figure 7 we show the extraction of the surface of the left ventricle from the 3D ultrasound image shown in figure 1. For this ultrasound image of size $256 \times 256 \times 256$ we used a personal computer with a $1.4 \mathrm{Ghz}$ processor and 512 $\mathrm{Mb}$ of RAM. The segmentation was obtained in less than 15 seconds.

\section{Conclusion}

In this paper we have presented a method that generalizes globally minimal paths for curve segmentation in 2D to surface segmentation in 3D. Our model is initialized by two user-supplied curves which we maximally exploit, partly by the fact 

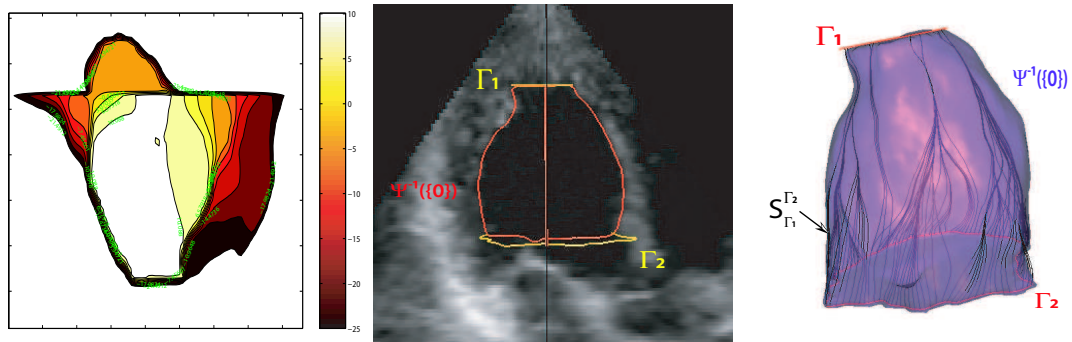

Figure 7: Left ventricle segmentation : on the left, some level sets of our solution $\Psi$ on a plane. middle shows the intersection of the zero level set of $\Psi$ with a slice of the image and on the right a volume representation of $\Psi^{-1}(\{0\})$.

that the surface we generate is constrained to contain them. We have developed a novel implicit approach that, through a linear partial differential equation, exploits the solution to the Eikonal equation and generates a function whose zero level set contains all the globally minimal paths between the constraining curves. Hence, our approach is not prone to local minima traps as are other active surface approaches.

\section{References}

[1] L. Ambrosio. Transport equation and Cauchy problem for BV vector fields. Preprints Scuola Normale Superiore, Department of Mathematics: http://cvgmt.sns.it/people/ambrosio/, 2003.

[2] R. Ardon and L.D. Cohen. Fast constrained surface extraction by minimal paths. 2nd IEEE Workshop on Variational, Geometric and Level Set Methods in Computer Vision, pages 233-244, October 2003.

[3] R. Ardon and L.D. Cohen. Fast Constrained Surface Extraction by Minimal Paths. To appear in International Journal of Computer Vision, 2006.

[4] F. Bouchut, F. James, and S. Mancini. Uniqueness and weak stability for multi-dimensional transport equations with one-sided lipschitz coefficient. Prépublications du département Mathématiques et Applications, Physique Mathématique d'Orléans, 2004.

[5] A.M. Bruckstein. On shape from shading. Computer Vision, Graphics and Image Processing, 44(2):139-154, November 1988. 
[6] M. P. Do Carmo. Differential Geometry of Curves and Surfaces. PrenticeHall, 1976.

[7] V. Caselles, R. Kimmel, and G. Sapiro. Geodesic active contours. International Journal of Computer Vision, 22(1):61-79, 1997.

[8] V. Caselles, R. Kimmel, G. Sapiro, and C. Sbert. Minimal-surfaces based object segmentation. IEEE Transactions On Pattern Analysis and Machine Intelligence, 19(4):394-398, April 1997.

[9] L.D. Cohen. Multiple contour finding and perceptual grouping using minimal paths. Journal of Mathematical Imaging and Vision, 14(3), 2001. Jan 2001.

[10] L.D. Cohen and R. Kimmel. Global minimum for active contour models: A minimal path approach. International Journal of Computer Vision, 24(1):5778, August 1997.

[11] T. Deschamps and L.D. Cohen. Fast extraction of minimal paths in 3D images and applications to virtual endoscopy. Medical Image Analysis, 5(4), December 2001.

[12] M. Kass, A. Witkin, and D. Terzopoulos. Snakes: Active contour models. International Journal of Computer Vision, 1(4):321-331, 1988.

[13] R. Kimmel and J.A. Sethian. Optimal algorithm for shape from shading and path planning. Journal of Mathematical Imaging and Vision, 14(3):237-244, 2001.

[14] C. Mantegazza and A. C. G. Mennucci. Hamilton-Jacobi equations and distance functions on Riemannian manifolds. Appl. Math. Opt., 47(1):1-25, 2003.

[15] A. C. G. Mennucci. Regularity and variationality of solutions to hamiltonjacobi equations. part i: regularity. ESAIM COCV, 10(1):426-451, july 2004.

[16] S. Osher and J.A. Sethian. Fronts propagating with curvature dependent speed: algorithms based on the hamilton-jacobi formulation. Journal of Computational Physics, 79:12-49, 1988.

[17] J.A. Sethian. A fast marching level set method for monotonically advancing fronts. Proceedings of the National Academy of Sciences, 93(4):1591-1595, February 1996. 
[18] J.A. Sethian. Level set methods: Evolving Interfaces in Geometry, Fluid Mechanics, Computer Vision and Materials Sciences. Cambridge University Press, University of California, Berkeley, 2nd edition, 1999.

[19] J. N. Tsitsiklis. Efficient algorithms for globally optimal trajectories. IEEE Transactions on Automatic Control, 40(9):1528-1538, September 1995.

[20] A. Yezzi and J. L. Prince. An Eulerian PDE Approach for Computing Tissue Thickness. IEEE Transactions On Medical Imaging, 22:1332-1339, October 2003. 